\title{
Focused Ion Beam Fabrication of Individual Carbon Nanotube Devices
}

Lee Chow ${ }^{1}$, and Guangyu Chai ${ }^{2}$

${ }^{1}$ Physics, University of Central Florida, 4000 Central Florida Blvd., Orlando, FL, 32816-2385

${ }^{2}$ Apollo Technologies Inc., Orlando, FL, 32750

\begin{abstract}
Focused ion beam (FIB) techniques have found many applications in nanoscience and nanotechnology applications in recent years. However, not much work has been done using FIB to fabricate carbon nanotube devices. This is mainly due to the fact that carbon nanotubes are very fragile and energetic ion beam from FIB can easily damage the carbon nanotubes. Here we report the fabrication of carbon nanotube (CNT) devices, including electron field emitters, atomic force microscope tips, and nano-pores for biomedical applications. This is made possible by a unique, coaxial configuration consisting of a CNT embedded in a graphitic carbon coating, which was developed by us for FIB processing of carbon nanotubes. The CNT-based atomic force microscope tip has been demonstrated. The electron field emission from the tip and the side wall of CNT will be discussed. We will also report the fabrication of a multiwall carbon nanotube nanopore for future applications.
\end{abstract}

\section{INTRODUCTION}

Since its discovery [1] in 1991, carbon nanotube (CNT) attracted intense interest because of their potential applications in nanotechnology [2-6]. All these applications are due to the unique properties of CNTs, among which are, high aspect ratio, large Young's modulus [7], chemical inertness, and excellent field emission properties [8]. There are many demonstrated and proposed applications of carbon nanotube devices. In general, we can classify them into two categories: applications that required large quantities of CNTs, and applications that only required one single CNT. Here in this paper, we will concentrate on the applications that only requires one single $\mathrm{CNT}$ in each device. In particular, the following three carbon nanotube devices will be considered: carbon nanotubes as electron sources for electron microscopes, carbon nanotubes as sharp probe tips for the atomic force microscope, and carbon nanotubes as nano-pore for biological applications.

Focused ion beam (FIB) $[9,11]$ is a very versatile technique to remove or to deposit materials. Because of its ability to focus an ion beam to a spot size of a few nanometers, FIB has found numerous applications [12] in nanofabrication. The focused ion beam technology is based on liquid metal ion source and ion optics technology developed in the late ' 70 and early ' 80 . The two most important functions of an FIB system are its ability to fabricate nanometer size structures and to deposit minute amounts of materials with nanometer precision.

Recently, researchers started to apply FIB techniques to CNT-related applications. The initial efforts involved applying FIB to modify and manipulate CNTs directly with Ga+ ion 
beams [13-16]. However, due to the high impact momentum of $\mathrm{Ga}^{+}$, the carbon atoms are easily knocked out from their lattice locations and structural defects were induced inside the CNTs.

These defects are unavoidable when CNTs are directly exposed to a $\mathrm{Ga}^{+}$ion beam, and weaken the electrical and mechanical properties of the CNTs which are essential for CNT applications. Since the CNT defects generated by FIB appeared to be inevitable, researchers started to use the defects on CNTs intentionally. Some promising applications included using FIB to open capped single-wall carbon nanotubes (SWNT) [17] and applying FIB to generate damaged areas on

SWNTs to fabricate quantum dots[18].

\section{EXPERIMENTAL PROCEDURE}

We have developed a chemical vapor deposition technique [19] to grow a fiber-protected carbon nanotubes (F-CNT). Our main goal was to grow a configuration of carbon nanotube that would be easy to handle and could withstand the intense ion beam in a focused ion beam instrument. We adopted the following strategy to accomplish the task. We first prepared $30 \mathrm{~nm}$ size catalysts on a silicon substrate using evaporation under Helium atomsphere. We then heated our substrate under a 50/50 mixture of $\mathrm{Ar}$ and $\mathrm{H}_{2}$ gases to $725^{\circ} \mathrm{C}$ to purge the growth chamber and to reduce the Fe/Ni nanoparticles to metallic form. We then turned off $\mathrm{H}_{2}$ gas, and add $10 \%$ methane with Ar and continued heating the substrate to $875^{\circ} \mathrm{C}$. During this stage, carbon nanotubes were grown on the silicon substrate by the catalytic vapor growth process. The length of the nanotube depended on the growth duration while the diameter of the nanotube is mainly controlled by the initial catalytic nanoparticle size. We then reduced the methane concentration to $5 \%$ of the total mixture and added $5 \%$ of $\mathrm{H}_{2}$ and increased the temperature to a level between 900 and $1000{ }^{\circ} \mathrm{C}$ for 10 minutes. This step was used to deposit a thin layer of amorphous carbon on the outside wall of the CNTs. Afterward, we increased the temperature to $1000^{\circ} \mathrm{C}$ for 30 minutes. During this final step, a graphitic carbon outer layer is formed on top of the thin amorphous carbon. In Figure 1, an SEM micrograph of the F-CNT is shown together with a schematic diagram of the F-CNT.
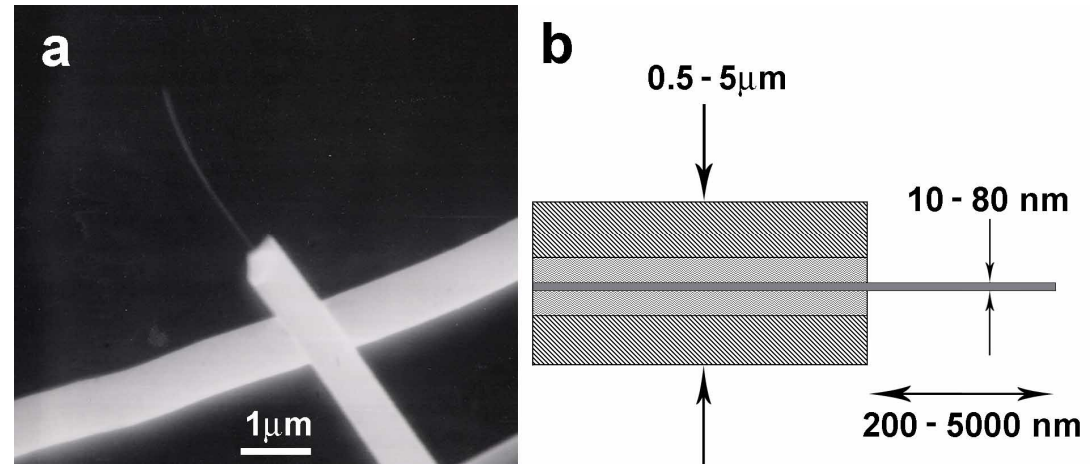

Figure 1. (a) SEM image of the as-grown fiber-protected carbon nanotube. (b) A schematic diagram of the fiber-protected carbon nanotube.

Once the F-CNTs were synthesized, the outer carbon layer was broken mechanically. The carbon nanotube is stronger than the carbon fiber, so it is possible to crush the outer carbon 
layer and reveal the inner CNT core as shown in Figure 1(a) above. This way, we end up with a co-axial nano-structure with the CNT as the core of micron size carbon fiber which can be easily manipulated with a micro-manipulator under an optical microscope. These F-CNTs were then transferred to FIB chamber using a clean tungsten wire with silver paint at the tip to glue these micron size fibers. It should be noted here that due to the minute size of carbon nanotubes, the nanotubes can be easily damaged by energetic ion beams. We observed a "softening" of the CNTs due to exposure to $\mathrm{Ga}^{+}$ion beams in our recent work [20]. It is very desirable to use a dual-beam FIB instrument for the processing of carbon nanotube devices for the above reason. However, our work reported below was done on a single beam FEI Vectra 200 FIB instrument.

\section{FIB fabrication of CNT atomic force microscope (AFM) tip}

A tungsten needle micro-manipulator installed inside the FIB chamber is used to assist the fabrication of the CNT AFM tip. We first use the micromanipulator to pick up an intermediate carbon fiber. This step is necessary to give us an extra degree of freedom to manipulate the CNT samples. For each "pick-up" procedure, we first use FIB milling to create a slot on the tungsten needle, we then move the manipulator and intermediate fiber together and deposit Pt metal at the joint to weld the fiber and tungsten needle together (see Figure 2(a)). Afterward, FIB is used to cut the intermediate fiber away from the substrate. The reason to mill a slot before each welding step is to increase the strength of the weld joint. Once we have the intermediate fiber welded on the manipulator, we then use the exact same procedure to pick up the desired fiber that contains the CNT at the end (see figure 2(b)). Next, we cut a deep slot at the tip of a conventional AFM cantilever pyramid with FIB, move the fiber into the slot, weld them together, and cut off the intermediate fiber from the AFM tip (See Figure 2(c)).

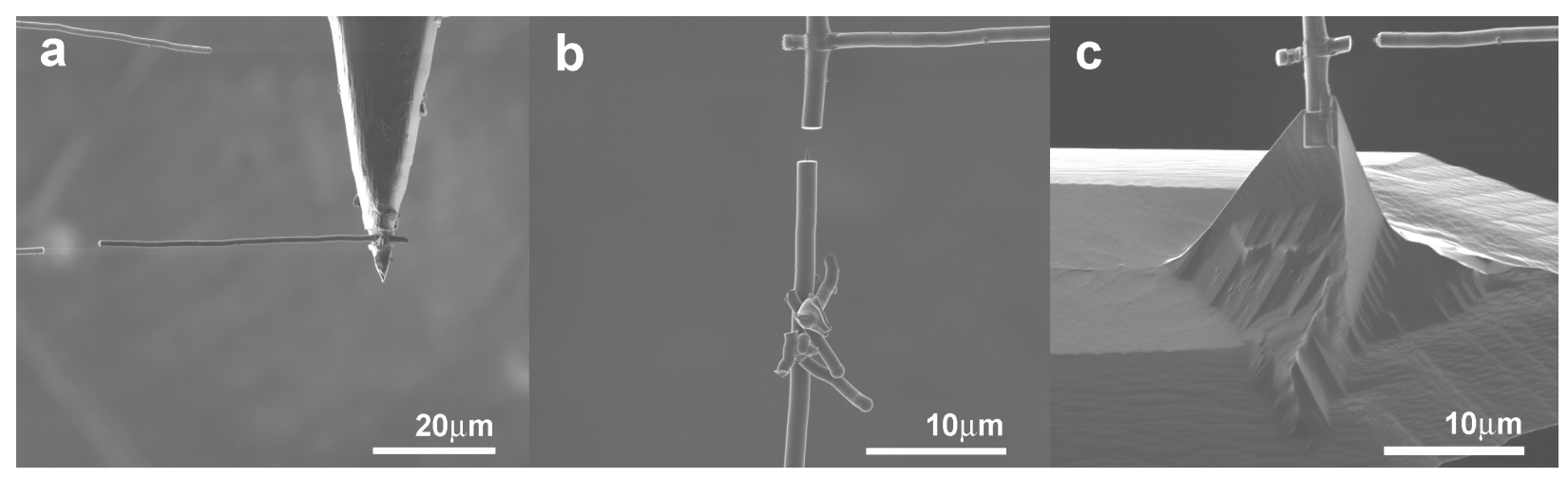

Figure 2. Secondary electron images of the FIB fabrication process. (a) Using a tungsten needle to pick up a carbon fiber as the intermediate fiber. (b) Using the intermediate fiber to pick up the desired fiber with nanotube at the end of the fiber. (c) Mounting the fiber on the pyramid tip of an AFM cantilever.

In figure 3 below, we show an SEM image of an AFM cantilever with a carbon nanotube positioned at the tip of the pyramid. As can be seen in the image, the micron size fiber with a 
nanotube tip is bonded to the top of the pyramid tip. The CNT tip is perfectly aligned vertically with the AFM cantilever. A close-up high magnification SEM image on the right shows that this carbon fiber has a diameter about $1.3 \mu \mathrm{m}$, the CNT radius is about $20 \mathrm{~nm}$, and the CNT length is about $150 \mathrm{~nm}$.

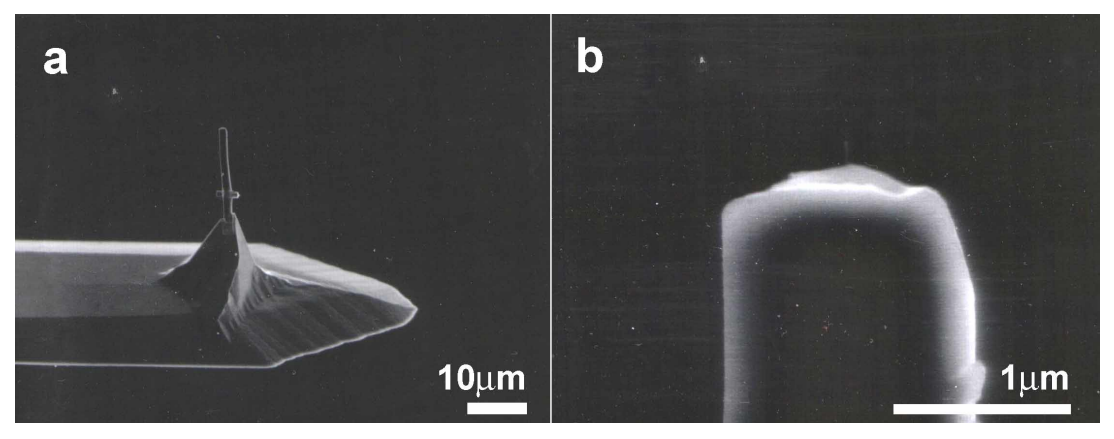

Figure 3. (a) The AFM cantilever with F-CNT tip mounted on the top of the pyramid. (b) A close-up image shows the carbon fiber with its nanotube core.

\section{FIB fabrication of CNT electron field emitter}

The fabrication of a CNT electron field emitter [21] is very similar to the fabrication of a CNT AFM tip. We start out with a $200 \mu$ m diameter tungsten wire and etch the tip down to a radius of less then $0.1 \mu \mathrm{m}$. Then we mounted the etched tungsten tip in the FIB instrument and used the FIB to mill a slot at the end of the tungsten wire. The same procedure described above is used to pick up the carbon fiber which contains the carbon nanotube and mount it on the tip of the tungsten wire as shown in Figure 4(a) below. In Figure 4(b) a close-up SEM image of the carbon nanotube is shown.

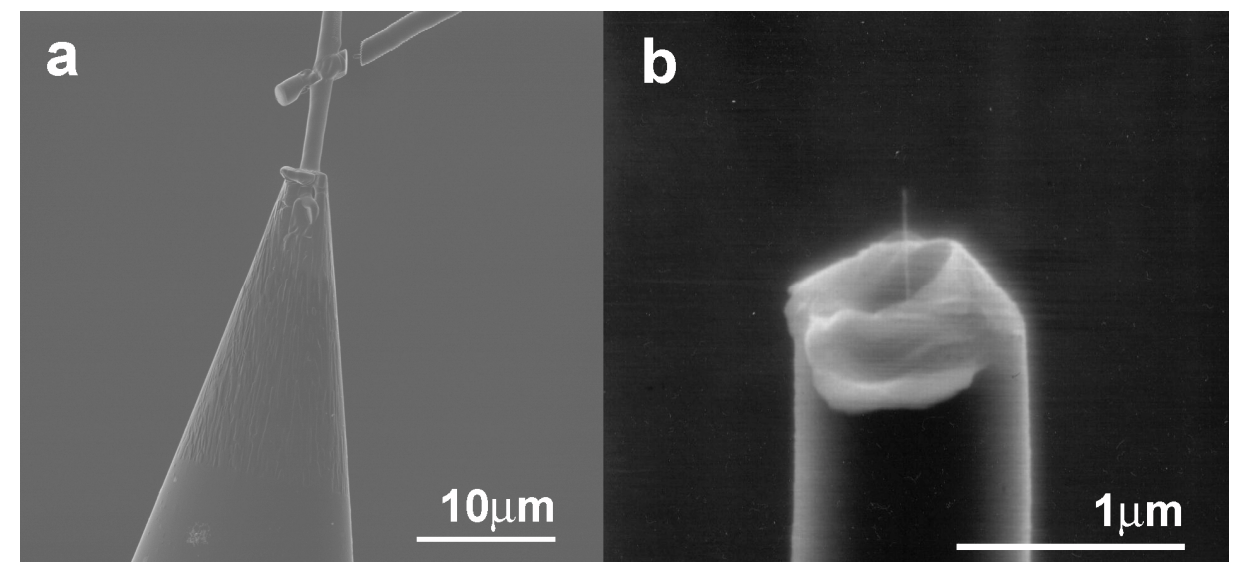

Figure 4. (a) Mounting a fiber-protected CNT on an etched W filament. (b) A close-up view of the tip of carbon fiber showing the CNT at the core of the carbon fiber. 
Since one of the major applications for a CNT field emitter will be to act as the electron source for a field emission electron microscope, we also demonstrated that it is possible to fabricate our carbon nanotube field emitter on a conventional tungsten filament SEM source. In figure 5, our carbon nanotube field emission tip is shown on a commercial tungsten filament. Here we used the FIB to fabricate a slot at the apex of the tungsten filament first, and then carbon fiber bearing the CNT tip was mounted in the slot and Pt metal was used to secure the joint, as in the procedure described earlier.

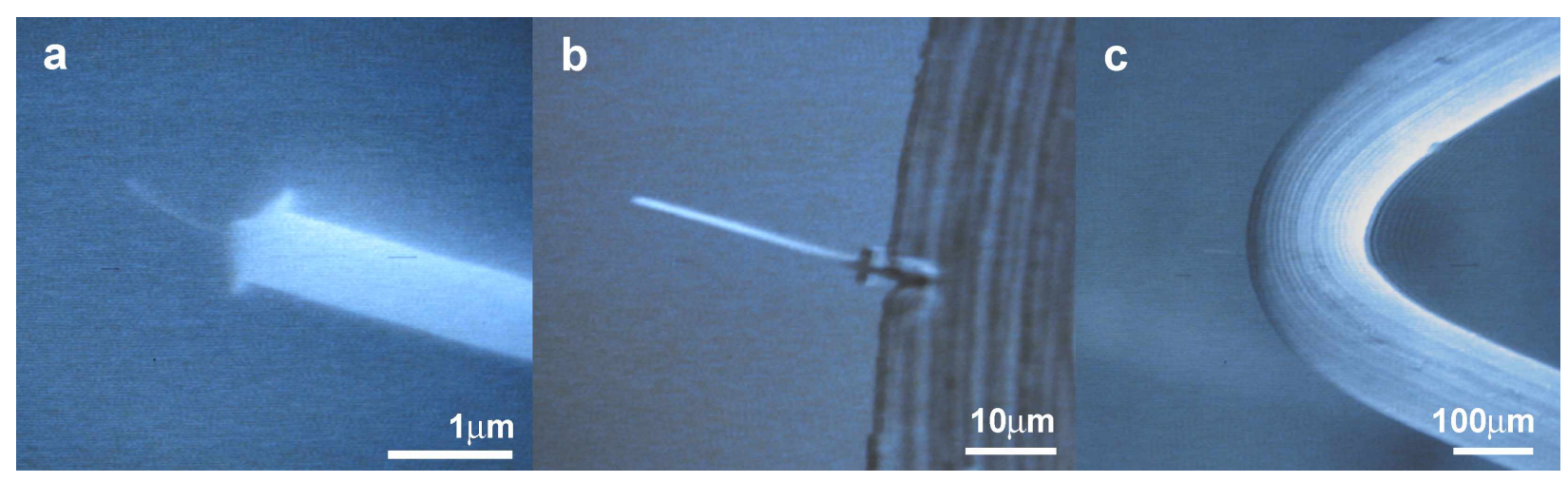

Figure 5. Secondary electron images of carbon nanotube fabricated using FIB on a conventional tungsten SEM electron source. (a) A close-up view of the carbon nanotube tip on the carbon fiber. (b) A broader view of the carbon fiber at the apex of the $\mathrm{W}$ filament. (c) A zoom-out view of $\mathrm{W}$ filament with the F-CNT barely seen.

\section{FIB fabrication of CNT nanoaperture/nanopore}

Another area of research that we started recently involves using focused ion beam to fabricate carbon nanotube nanoaperure/nanopore. In 2001, Meller et al [22] demonstrated that DNA can be driven through a nanopore with an applied voltage. The authors used a biological nanopore: $\alpha$-hemolysin. Although biological nanopores are useful, there are a lot of advantages to use solid state nanopores [23-25], such as stability and controllability. In 2002, Siwy et al [26] used a track etching technique to produce a synthetic nanohole on a membrane. The intense interest in nanopores are due to the possibility of using nanopores as a sequencing tool for DNA molecules.

To fabricate a CNT nanopore, we started out with our fiber-protected carbon nanotube lying on a silicon substrate. First, a stripe of Pt metal about $3 \mu \mathrm{m}$ x $20 \mu \mathrm{m}$ was deposited perpendicular to the length of carbon nanotube to protect and secure the carbon fiber. We then used the standard TEM cross-sectional sample preparation technique [27] to lift a cross-section of carbon nanotube and silicon sample. This sample is the size of a typical TEM sample, so it is not difficult to handle. The sample is directly transferred to a TEM grid inside the FIB chamber for future measurements.

In Figure 6(a), a fiber-protected carbon nanotube lies flat on the silicon substrate is shown. In Figure 6(b), the lift-off procedure is half completed in this image. We can see that the Pt metal has been deposited and two large cavities have been milled on both sides of the sample. 
In Figure 6(c), the sample has been transferred from the silicon substrate and mounted on a predrilled slot on the side of a copper TEM grid. Note that the magnification in this image has been reduced by a factor of 10 , so the sample appears smaller.

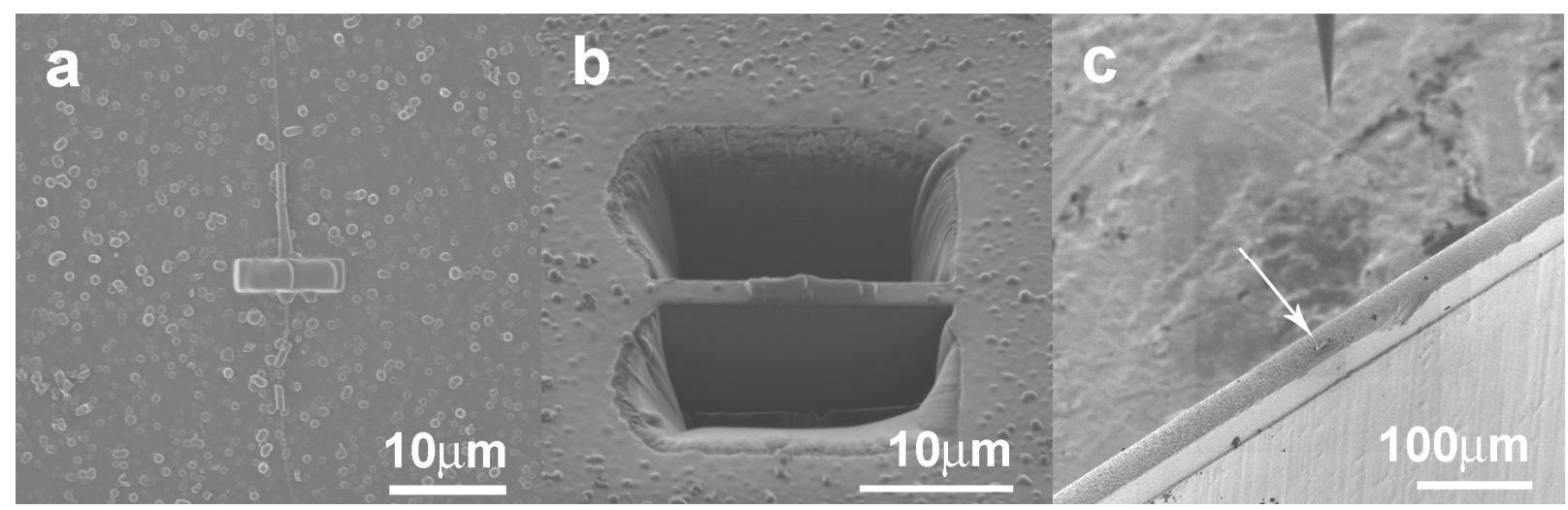

Figure 6. (a) A fiber-protected carbon nanotube with a stripe of Pt metal deposited over it. (b) Standard TEM sample preparation technique is used to lift off a cross-section sample from the substrate. (c) Use the in-situ micromanipulator to transfer the sample to a $\mathrm{Cu}$ TEM grid for further testing under TEM. The arrow indicates the position of the sample.

The possible exponential improvement in the quantum computation speed has spark intense interest in quantum computer [28]. One possible realization of a solid state quantum computer [29] would be an array of ${ }^{31} \mathrm{P}$ atoms embedded in an isotopically enriched ${ }^{28} \mathrm{Si}$ substrate. To implement the above idea, single ion implantation with nanometer accuracy is required. We want to note here that the CNT nanopore sample prepared above can be used as a nano-aperture for single ion implantation [30-31], or as a channeling device for charged particles [32].

\section{RESULTS and DISCUSSION}

\section{CNT atomic microscope tip}

The CNT-tipped AFM cantilever fabricated is then mounted into a Nomad scanning probe microscope from Quesant Instruments for testing. A standard optical interference grating pattern is used as a sample for the comparison between our CNT tip and a commercial silicon tip. The results are shown in Figure 7. In Figure 7(a), the image was obtained using our CNT tip, while in Figure 7(b), a new silicon tip was used. Here we can see that the image from CNT tip indeed showed much more details and the size of the grains were much smaller. The image scanned using a new silicon tip in Figure 7(b) showed a much coarser grain size. 


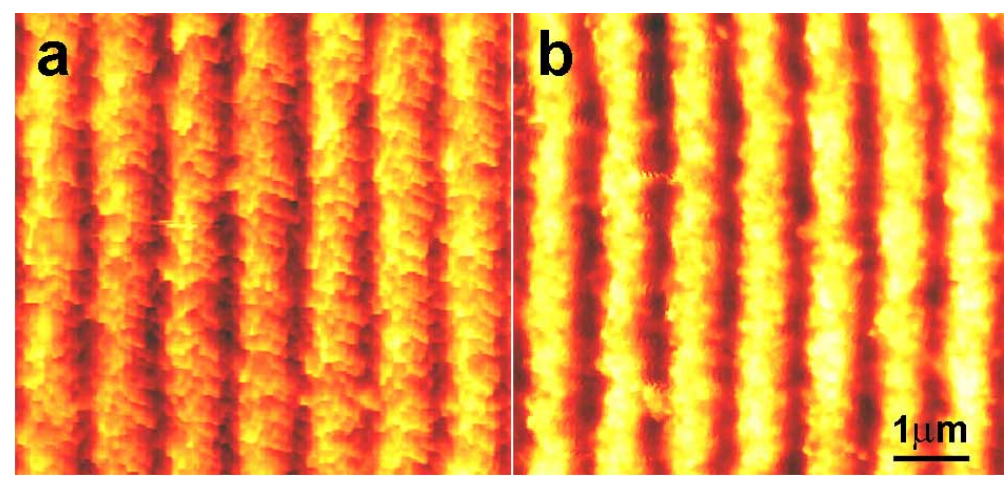

Figure 7. (a) Image scanned by a CNT AFM tip fabricated with focused ion beam. (b) Image scanned by a conventional silicon AFM tip.

In addition to better spatial resolution, a CNT AFM tip has the advantages of longer operating lifetime and the ability to image the bottom of a narrow trench [2]. A typical conventional silicon AFM tip can only last for about 30 minutes before the tip starts to wear out, while a CNT-based scanning probe tip can last much longer as shown in Figure 8 below.

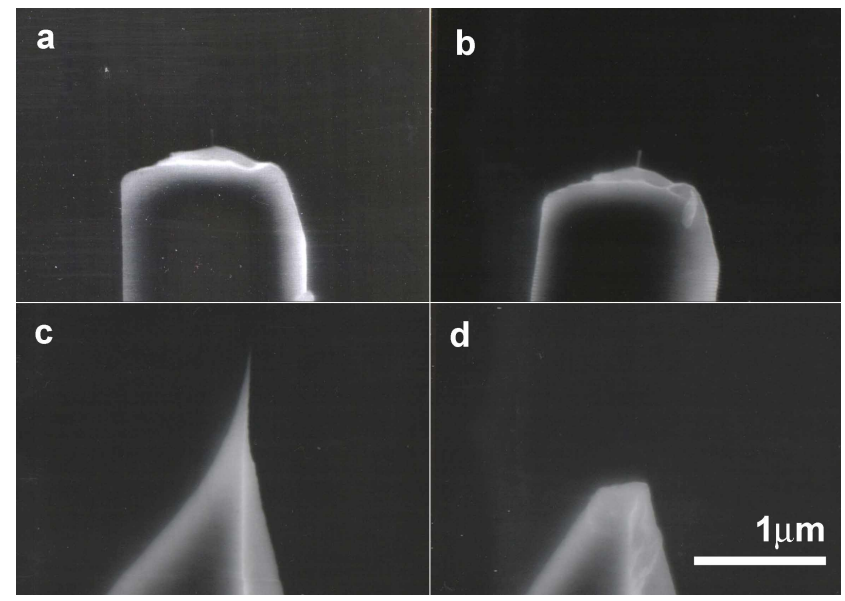

Figure 8. (a) CNT before scan. (b) CNT tip after $2 \mathrm{hr}$ of scan. (c) Si tip before scan. (d) Si tip after 0.5 hour of scan.

\section{Current voltage characteristic of a CNT electron field emitter}

Individual CNT-based electron field emitters fabricated by FIB techniques were tested in an ultrahigh vacuum chamber [20,21]. We found that the I-V characteristic curve of the field emission followed the standard Fowler-Nordheim relationship with a turn-on voltage of 120 volts. A typical I-V curve and a Fowler-Nordheim plot of the electron emission from the CNT tip are shown in Figure 9 below. 

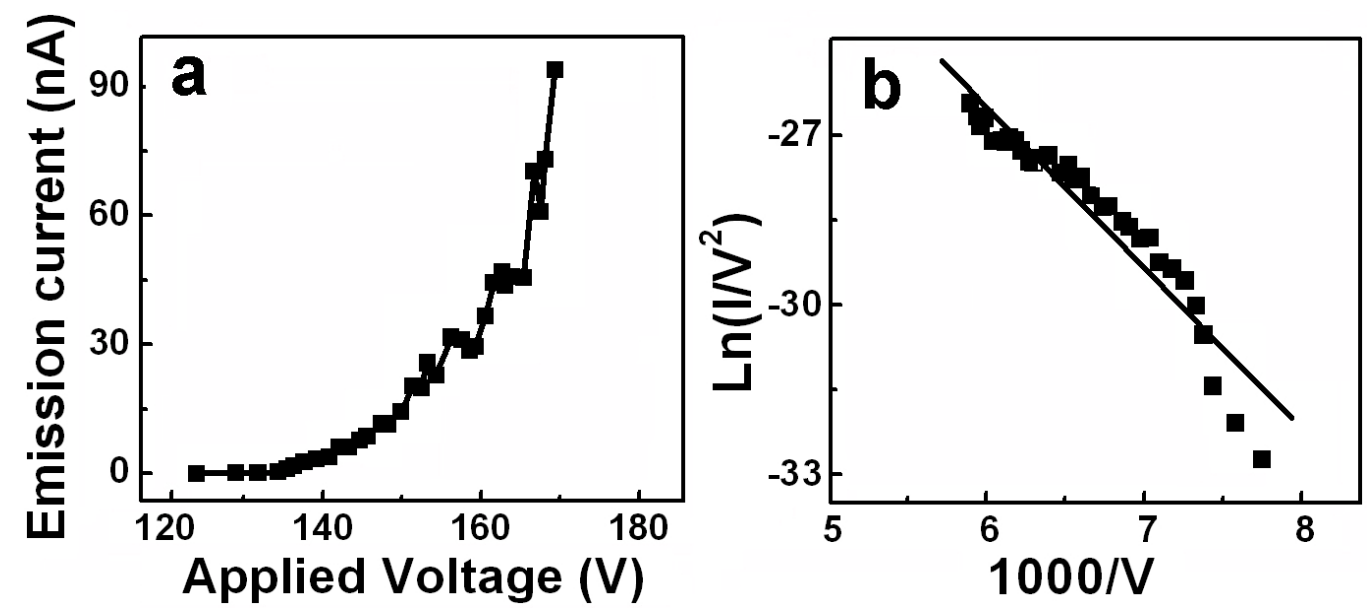

Figure 9. (a) A typical I-V characteristic curve of CNT field emitter. (b) The semi-logarithmic plot shows the Fowler-Nordheim relationship.

\section{TEM testing of the nanopore sample}

To check the nanometer size channel of the carbon nanotube sample in Figure 6(c), we used a FEI Tecnai F30 scanning transmission electron microscope. We employed a $120 \mathrm{KeV}$ unfocused electron beam and a CCD detector behind the sample to detect electrons passed through the hollow core of CNT. In Figure 10 below, we can see clearly the image produced by electrons channel through the core of carbon nanotube.

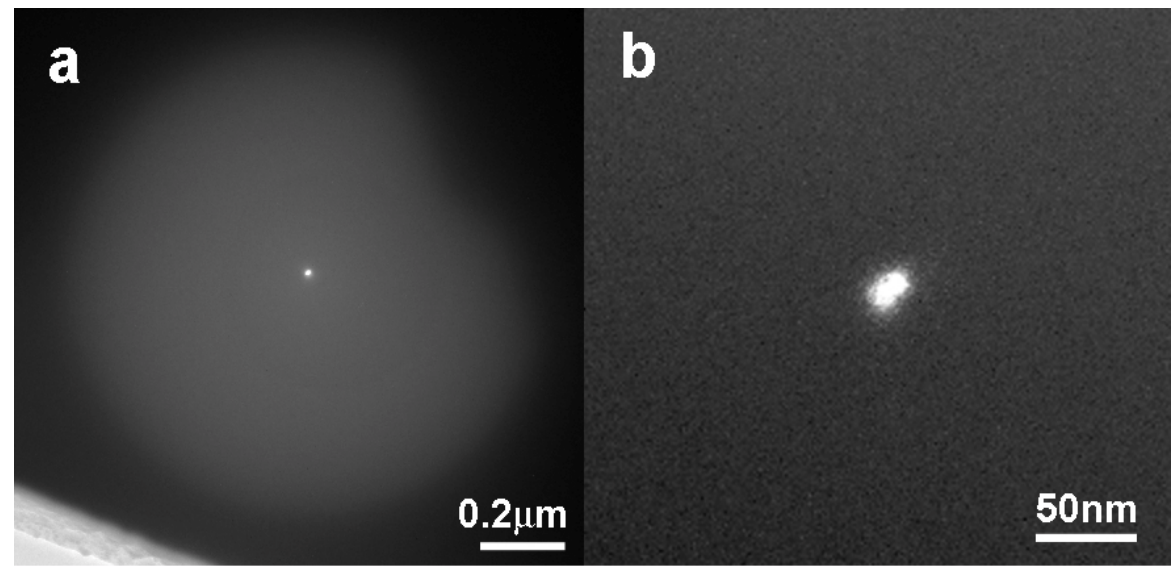

Figure 10. (a) Image of the electrons channel through the CNT hollow core detected by a CCD detector. (b) A magnified image of electrons channel through CNT hollow core. The diameter of the hole is about $12-15 \mathrm{~nm}$. 


\section{CONCLUSIONS}

In conclusion, in this paper we demonstrated that focused ion beam techniques can be used to fabricate carbon nanotube nano-devices. The FIB fabrication process is aided by the synthesis of a unique fiber-protected carbon nanotube configuration. Three different types of CNT-based nano-devices were demonstrated. We showed that CNT AFM tips can be fabricated with FIB. The device has the advantages of a large aspect ratio and a significantly longer operating lifetime. We also showed that F-CNT electron filed emitter can be fabricated with FIB method. These CNT-based electron field emitters can be used as electron sources in a field emission electron microscope. We also demonstrated the fabrication of nanopores based on carbon nanotubes. The nanopore we constructed has an inner diameter of $12 \mathrm{~nm}$ and the length of the nanopore can be adjusted.

\section{ACKNOWLEDGMENTS}

The authors would like to acknowledge the contributions from our past and current collaborators: Dr. Dan Zhou, Dr. S. Kleckley, Dr. R. Vanfleet, Dr. Weili Luo, Dr. H. Heinrich, and Dr. T. Schenkel. We also acknowledge Dr. Ted Tessner of FEI company for providing the tungsten filament used in figure 4. Technical support from the staff of UCF's Material Characterization facility and partial financial support from Apollo Tech, Inc. is greatly appreciated.

\section{REFERENCES}

1. S. Iijima, Nature 354, 56 (1991).

2. H. Dai, J. H. Hafner, A. G. Rinzler, D. T. Colbert, and R. E. Smalley, Nature 384, 147 (1996).

3. W. A. De Heer, A. Chatelain, and D. Ugarte, Science, 2701179 (1995).

4. S. J. Trans, A. R. M. Verschuren, and C. Dekker, Nature 393, 49 (1998).

5. P. G. Collins and A. Zettl, Appl. Phys. Lett. 69, 1969 (1996).

6. A. Bachtold, P. Hadley, T. Nakanishi, and C. Dekker, Science, 294, 1317 (2001).

7. M. M. J. Treacy, T. W. Ebbesen, and J. M. Gibson, Nature, 381, 678 (1996).

8. G. Rinzler, J. H. Hafner, P. Nikolaev, L. Lou, S. G. Kim, D. Tomanek, P. Nordlander, D. T. Colbert, and R. E. Smalley, Science, 269, 1550 (1995).

9. J. Orloff, M. Utlaut, and L. Swanson, "High Resolution Focused Ion Beams", Kluwer Academic/Plenum Publishers, New York, (2003).

10. L. A. Giannuzzi and F. Stevie, "Introduction to Focused Ion Beams: Theory, Instrumentation, Applications, and Practice", Springer, NY (2005).

11. Wagner, J. P. Levin, J. L. Mauer, P. G. Blauner, S. J. Kirch, and P. Longo, Journal of Vacuum Science \& Technology B, 8, 1557 (1990).

12. A. A. Tseng, Small, 1924 (2005).

13. M. S. Raghuveer, P. G. Ganesan, J. D Arcy-Gall, and G. Ramanath, Appl. Phys. Lett., 84,4484 (2004). 
14. Y.J. Jung, Y. Homma, R. Vajtai, Y. Kobayashi, T. Ogino, and P. M. Ajayan, Nano Letters, 4, 1109 (2004).

15. Z. Deng, E. Yenilmez, A. Reilein, J. Leu, H. Dai, and K. A. Moler, Appl. Phys. Lett., 88, 023119 (2006).

16. C. S. Han, J. K. Park, Y. H. Yoon, and Y. H. Shin, Carbon, 44, 3348 (2006).

17. M. J. Kim, E. Haroz, Y. Wang, H. Shan, N. Nicholas, C. Kittrell, V. V. Moore, Y. Jung, D. Luzzi, R. Wheeler, T. BensonTolle, H. Fan, S. Da, W. Hwang, T. J. Wainerdi, H. Schmidt, R. H. Hauge, and R. E. Smalley, Nano Letters, 7, 15 (2007).

18. K. Maehashi, H. Ozaki, Y. Ohno, K. Inoue, K. Matsumoto, S. Seki, and S. Tagawa, Appl. Phys. Lett., 90, 023103 (2007).

19. S. Kleckley, G. Y. Chai, D. Zhou, R. Vanfleet, and L. Chow, Carbon, 41833 (2003).

20. G. Chai and L. Chow, Carbon, 45, 281 (2007).

21. G. Chai, L. Chow, D. Zhou, and S. P. Byahut, Carbon 43, 2083 (2005).

22. A. Meller, L. Nivon, and D. Branton, Phys. Rev. Lett. 86, 3435 (2001).

23. C. Schmidt, , M.Mayer, and H. Vogel, Angew. Chem. Int. Edn, 39, 3137 (2000).

24. N. Fertig, et al., Phys. Rev. E 64, 040901 (2001).

25. C. Dekker, Nature Nanotechnology, 1, to appear. (2007).

26. Z. Siwy and A. Fulinski, Phys. Rev. Lett. 89, 198103 (2002).

27. Yamaguchi, M. Shibata, and T. Hashinaga, Journal of Vacuum Science \& Technology B, 11, 2016 (1993).

28. C. H. Bennett and D. P. DiVincenzo, Nature 404, 247 (2000).

29. B. E. Kane, Nature 393, 133 (1998).

30. T. Schenkel, A. Persaud, S. J. Park, J. Nilsson, J. Bokor, J. A. Liddle, R. Keller, R. H. Schneider, D. W. Cheng, and D. E. Humphries, J. Appl. Phys. 94, 7017 (2003).

31. T. Schenkel, V. Radmilovic, E. A. Stach, S. J. Park, and A. Persaud, J. Vac. Sci. \& Tech. B 21, 2720 (2003).

32. A. V. Krasheninnikov and K. Nordlund, Phys. Rev. B 71, 245408 (2005). 